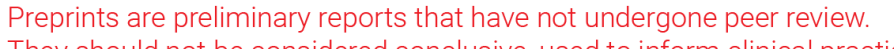 They should not be considered conclusive, used to inform clinical practice, or referenced by the media as validated information. \\ Leukocyte telomere length and amyotrophic lateral sclerosis: a Mendelian randomization study
}

\section{Kailin Xia}

Peking University Third Hospital

\section{Linjing Zhang}

Peking University Third Hospital

\section{Gan Zhang}

Peking University Third Hospital

\section{Yajun Wang}

Peking University Third Hospital

\section{Tao Huang}

Peking University School of Public Health

Dongsheng Fan ( $\nabla$ dsfan2010@aliyun.com)

Peking University Third Hospital https://orcid.org/0000-0002-3129-9821

\section{Research Article}

Keywords: amyotrophic lateral sclerosis, leukocyte telomere length, Mendelian randomization, risk factors

Posted Date: October 1st, 2021

DOI: https://doi.org/10.21203/rs.3.rs-522773/v2

License: (c) (1) This work is licensed under a Creative Commons Attribution 4.0 International License. Read Full License

Version of Record: A version of this preprint was published at Orphanet Journal of Rare Diseases on December 1st, 2021. See the published version at https://doi.org/10.1186/s13023-021-02135-2. 


\section{Abstract \\ Background}

Observational studies have suggested that telomere length is associated with amyotrophic lateral sclerosis (ALS). However, it remains unclear whether this association is causal. We employed a twosample Mendelian randomization (MR) approach to explore the causal relationship between leukocyte telomere length (LTL) and ALS based on the most cited and most recent and largest LTL genome-wide association studies (GWASs) that measured LTL with the Southern blot method $(n=9190)$ and ALS GWAS summary data $(n=80,610)$. We adopted the inverse variance weighted (IVW) method to examine the effect of LTL on ALS and used the weighted median method, simple median method, MR Egger method and MR PRESSO method to perform sensitivity analyses.

\section{Results}

We found that genetically determined longer LTL was inversely associated with the risk of ALS (OR = $0.846,95 \% \mathrm{Cl}: 0.744-0.962, \mathrm{P}=0.011)$, which was mainly driven by rs 940209 in the OBFC1 gene, suggesting a potential effect of OBFC1 on ALS. In sensitivity analyses, that was confirmed in MR Egger method $(\mathrm{OR}=0.647,95 \% \mathrm{Cl}=0.447-0.936, \mathrm{P}=0.050)$, and a similar trend was shown with the weighted median method $(\mathrm{OR}=0.893, \mathrm{P}=0.201)$ and simple median method $(\mathrm{OR}=0.935 \mathrm{P}=0.535)$. The MR Egger analyses did not suggest directional pleiotropy, showing an intercept of $0.025(P=0.168)$. Neither the influence of instrumental outliers nor heterogeneity was found.

\section{Conclusions}

Our results suggest that genetically predicted longer LTL has a causal relationship with a lower risk of ALS and underscore the importance of protecting against telomere loss in ALS.

\section{Background}

Amyotrophic lateral sclerosis (ALS) is a deadly neurodegenerative disease that is considered to be an accelerated ageing disease [1]. Previous studies based on 10 countries and regions showed that the prevalence of ALS increases with ageing of the population [2]. However, the causes of ALS are largely unknown, although several possible etiopathogenesis mechanisms are currently being proposed. Therefore, identifying the causal factors for ALS may provide potential prevention strategies.

Leukocyte telomere length (LTL), known as the "molecular clock", is highly correlated with neurodegenerative diseases, cognitive function, and other age-related physiological indexes $[3,4]$. It has been shown that shortening telomeres by 1 standard deviation (SD) increases the risk of disease by $4 \%$ (log-odds ratio(OR) $=0.04$ per SD decrease in telomere length; $95 \% \mathrm{Cl}: 0.01-0.08, \mathrm{P}=0.01$ ) [5]. However, 
previous observational studies regarding telomere length and ALS are not always consistent. A study including 1,251 European patients with ALS revealed that telomeres were $9 \%$ longer in patients than in controls ( $95 \% \mathrm{Cl}: 3 \%-15 \%, \mathrm{P}=0.008)$ [6], but De Felice et al. [7] reported that the telomere length of 50 patients with sporadic ALS was significantly reduced by $15 \%$ compared with that of 50 healthy subjects. Furthermore, shorter telomeres were associated with earlier disease onset in animal models [8]. Due to the limits in selection bias, interference of confounders and the reverse causal relationships of observational studies, the causality between LTL and ALS remains largely ambiguous.

Mendelian randomization (MR) is a method for analyzing the causal relationship between exposure and an outcome by using genetic variations as instrumental variables (IVs) for exposure [9]. It is a promising statistical approach to overcome the limitations of observational studies and is similar to randomized controlled trials, in which risk alleles are naturally grouped to make powerful controls for reverse causality and confounders [10].

Therefore, in the present study, we employed two-sample MR analysis to explore the causal association between LTL and ALS. We selected two sets of instrumental variables from two separate genome-wide association studies (GWASs); one was the most cited in high-quality MR studies on LTL and the largest GWAS that measured LTL via the Southern blot method, while the other was the most recent and largest GWAS on LTL.

\section{Methods}

\section{GWAS summary data collection and IVs selection}

We searched PubMed for GWASs of LTL. From among these studies, we chose the studies with the most citations in Mendelian randomization studies (up to 12.2020) and the most recent publication including the largest sample size. IVs identified from the former were IV-1, while the other was IV-2.

The IV-1 was derived from a meta-analysis based on 6 studies, including 9190 European individuals (aged 18-95) [11]. Telomere length was a continuous variable measured with the Southern blot method for the terminal restriction fragment, which is the current gold standard for LTL measurement $[12,13]$. This is also the most recent and largest GWAS that adopted this method. During the meta-analysis, age, sex, and smoking were adjusted, and the mean telomere length was $6.83 \pm 0.65(\mathrm{~kb})$ (mean $\pm \mathrm{SD})$ in this study.

IVs were collected from the method most used in Mendelian randomization focused on LTL, the details and the quality of which have been described by Haycock et al. and testified in large scale MR estimates for many times $[3,4,14]$. Briefly, they were SNPs associated with LTL at the genome-wide significance level, whose effects and standard errors on LTL were concatenated by Mangino et al. [11]. Sixteen single nucleotide polymorphisms (SNPs) within the range of 10 loci were included after excluding loci with obvious heterogeneity between studies, which could explain $9.4 \%$ of the genetic variation in LTL. The statistical F value was 18-28 for each SNP [11] (displayed in Additional File supplementary table 1 in 
detail). It is considered an indicator for strong instruments and the absence of bias from weak instruments when the statistical value is greater than 10 [15].

We obtained IV-2 from the most recent and largest GWAS of LTL, which included up to 78,592 European participants from the EPIC-InterAct, EPIC-CVD and ENGAGE Consortium. They measured the mean LTL as a continuous variable by quantitative PCR which expressed LTL as a ratio of the telomere repeat number (T) to a single-copy gene (S) [16]. The age of participants ranged from 18 to 106 , which was adjusted in this GWAS as well as sex. Twenty SNPs were reported to be associated with ALS with $\mathrm{P}<5^{\star} 10^{-8}$ corrected by FDR, which were capable of explaining $1 \%-2 \%$ of the genetic variation in LTL. The F statistic of each SNP ranges from 27 to 205 (displayed in Additional File supplementary table 1 in detail).

To acquire outcome data from the same race, we used summary data from the most recently published ALS GWAS that genotyped and imputed more than 10 million SNPs in up to 20,806 ALS cases and 59,804 controls. All the patients had onset of symptoms after age 18 years and were diagnosed at probable or definite levels according to the El Escorial criteria [17].

We selected independent SNPs as IVs with $\mathrm{r}^{2}<0.001$ and MAF $>0.05$. For SNPs that could not be found in the ALS GWAS summary data, we replaced them with proxy SNPs in strong linkage disequilibrium (LD) $\left(r^{2}>0.9\right)$ by searching the SNiPA website(http://snipa.helmholtz-muenchen.de/snipa3/). If a proxy SNP was not reported, the SNP was excluded from downstream MR analysis. Thus, we obtained 10 independent SNPs in IV-1 and 15 SNPs in IV-2. The brief procedures are shown in the flowchart. (Additional File Supplementary Fig. 1).

\section{Two-sample MR}

According to IV-1 and IV-2, we extracted information including the effect allele, the other allele, effect, standard error, and P value of the corresponding SNPs from ALS summary data. We harmonized the direction of SNP effects on LTL and ALS.

MR analysis is based on the following 3 assumptions: assumption 1, the selected genetic variations are significantly associated with exposure; assumption 2 , the selected genetic variations are not associated with other confounders; and assumption 3, the selected genetic variations are significantly associated with the risk of outcome only through the pathway from exposure [18].

For MR, we implemented the multiplicative random effects inverse variance weighted (IVW) method as the main approach to examine the overall causal relationship between exposure and ALS based on the effect of SNPs on LTL and the effect of SNPs on ALS [19]. To validate the results from the IVW method, we applied the weighted median method, simple median method [20], MR Egger method [21] and MRPRESSO method as sensitivity analyses. To test potential pleiotropy, the MR Egger method, which is capable of reminding the presence of pleiotropy when the intercept significantly deviates from the origin, and MR-PRESSO analysis, which was used to detect the influence of outliers [22], were employed. The heterogeneity of SNPs used in IVW estimates was tested by Cochran's Q test, which suggests the 
presence of heterogeneity when it is lower than the significant $P$ value. Leave-one-out analysis and single SNP analysis were employed to evaluate the robustness of the significant results and the possibility of results being driven by a single SNP. We also calculated F statistics for IVs to demonstrate their strength. We performed the MR Steiger method to explore the potential reverse causal impact of ALS on the exposure [23]. We adopted a publicly available online tool to calculate the statistical power of our analysis (https://shiny.cnsgenomics.com/mRnd/). All the analyses were performed in R software version 3.6.3 [24]. The valid positive $P$ value was less than $0.025(0.05 / 2)$ after Bonferroni correction.

\section{Results}

In our study, two sets of LTL-related proxies were included to investigate the relationship between LTL and ALS using the five MR methods; detailed results are shown in Table 1 and the main results are visualized in Fig. 1. 
Table 1

Summary of the causal effects of each trait on ALS via different MR methods.

\begin{tabular}{|c|c|c|c|}
\hline & & IV-1 & IV-2 \\
\hline \multicolumn{2}{|l|}{ NSNPS } & 10 & 15 \\
\hline \multicolumn{2}{|l|}{ F statistics } & 954.49 & 1278.92 \\
\hline \multirow[t]{2}{*}{ Simple median } & OR $(95 \% \mathrm{Cl})$ & $0.935(0.756,1.156)$ & $0.981(0.774,1.242)$ \\
\hline & $P$ value & 0.535 & 0.872 \\
\hline \multirow[t]{2}{*}{ Weighted median } & OR $(95 \% \mathrm{Cl})$ & $0.893(0.750,1.062)$ & $0.982(0.786,1.227)$ \\
\hline & $P$ value & 0.201 & 0.872 \\
\hline \multirow[t]{2}{*}{ MR Egger } & OR $(95 \% \mathrm{Cl})$ & $0.647(0.447,0.936)$ & $0.839(0.520,1.352)$ \\
\hline & $P$ value & 0.050 & 0.483 \\
\hline \multirow[t]{2}{*}{ Inverse variance weighted } & OR $(95 \% \mathrm{Cl})$ & $0.846(0.744,0.962)$ & $0.941(0.797,1.111)$ \\
\hline & $P$ value & 0.011 & 0.471 \\
\hline \multirow[t]{2}{*}{ MR Egger } & intercept & 0.025 & 0.006 \\
\hline & $P$ value & 0.168 & 0.624 \\
\hline \multirow[t]{2}{*}{ Cochran's Q } & Q & 5.176278 & 12.014047 \\
\hline & $p$ value & 0.82 & 0.61 \\
\hline \multirow[t]{3}{*}{ MR-PRESSO } & RSSobs & 7.307 & 13.578 \\
\hline & $P$ value & 0.774 & 0.632 \\
\hline & outlier-corrected & NA & NA \\
\hline MR Steiger & $P$ value & 0.0002 & 0.023 \\
\hline \multicolumn{2}{|l|}{ Statistical power } & 0.6 & 0.2 \\
\hline
\end{tabular}

When we adopted the two-sample MR analysis based on the most cited genetic variants in LTL(IV-1), a longer LTL was inversely associated with the risk of ALS, which was alleviated by $15.4 \%$ (OR $=0.846,95 \%$ Cl: $0.744-0.962, P=0.011)$ for a genetically predicted one standard deviation (1-SD) increase in LTL via the IVW method. This causal association was confirmed with the MR Egger method $(\mathrm{OR}=0.647,95 \% \mathrm{Cl}=$ $0.447-0.936, P=0.050)$. The estimates based on the weighted median method ( $\mathrm{OR}=0.893,95 \% \mathrm{Cl}$ : $0.750-1.062, \mathrm{P}=0.201)$ and simple median method $(\mathrm{OR}=0.935,95 \% \mathrm{Cl}: 0.756-1.156, \mathrm{P}=0.535)$ showed similar trends but without significance. The MR Egger intercept showed no evidence of directional pleiotropy (intercept $=0.025, P=0.168$ ). There was no influence of instrumental outliers according to the MR-PRESSO analysis. Cochran's Q test indicated no heterogeneity. The MR Steiger test indicated that the causal direction of LTL IV-1 to ALS was in the right direction $(P<0.001)$. The scatter plots indicated the 
estimated effect of LTL on ALS by every single SNP (Fig. 2a). Through single SNP analysis, we found that this positive effect was mainly driven by rs9420907-C ( $O R=0.706, P=0.013)$ (Additional File Supplementary Fig. 2a), which was replicated in the leave-one out analysis (Additional File Supplementary Fig. 2b).

There was little evidence that longer LTL was associated with decreased risk of ALS in results produced with IVs acquired from the most recent and largest GWAS (IV-2). The OR of ALS per genetically predicted a 1-SD increase in telomere length was $0.941(95 \% \mathrm{Cl}: 0.797-1.111, \mathrm{P}=0.471)$ with the IVW method, which is similar to the sensitivity analyses (Table 1). No obvious horizontal pleiotropy interference was detected by the MR Egger intercept (intercept $=0.006, P=0.624$ ). Neither the influence of instrumental outliers nor heterogeneity was found. However, a significant impact of rs9419958-T on ALS was highly suggested in the single SNP analysis $(\mathrm{OR}=0.466, \mathrm{P}=0.015)$ (Supplementary Fig. 3 ).

We estimated the linkage disequilibrium (LD) of rs9420907 and rs9419958 using a publicly available online tool (http://snipa.helmholtz-muenchen.de/snipa3/index.php). They were both localized at the OBFC1 locus with $r^{2}$ equal to 1 , indicating that they are strongly linked.

\section{Discussion}

In the present study, we found that a longer LTL may be a protective factor for ALS in European population using two-sample MR method. Given the tight relationship between LTL and ageing, the results support the long-held view that ageing is associated with ALS [25]. Our findings suggest that LTL may participate in the pathogenesis of ALS and have clinical value for the prediction of ALS. The deceleration of LTL loss may be a breakthrough for the treatment of ALS in the future.

We evaluated the relationship between LTL and ALS through 2 different sets of IVs. We found that in the most commonly used genetic variance (IV- 1$)$, the risk of ALS was reduced by $15.4 \%$ (OR $=0.846,95 \% \mathrm{Cl}$ : $0.744-0.962, P=0.011$ ) for every genetically predicted $1-S D$ increase in the LTL. It was initially reported that 1-SD represented approximately 650 base pairs in LTL [11], which is equivalent to the loss of the natural ageing every 26 years in the European ancestor population [26]. In genetic variances extracted from the most recent and largest GWAS (IV-2), this trend seemed weak (OR $=0.941,95 \%$ Cl: 0.797-1.111, $P=0.471)$. Nevertheless, we relied more on the results from IV-1 for two reasons. First, the GWAS contributing to IV-1 was considered to be of higher quality. The methodology adopted for measuring LTL was Southern blot, which is the gold standard for LTL measurement [13]. The corresponding method in IV-2 was quantitative-PCR, which showed large variations among laboratories and only provided average telomere length as a relative ratio [27]. Second, the statistical power for analysis based on IV-1 was $40 \%$ higher than that based on IV-2 (Table 1). Thus, the consequence calculated from IV-1 was treated as the main one in our research and confirmed the studies indicating a protective effect of LTL on ALS. Notably, a similar article recently published showed that LTL had no direct causal effect on ALS and suggested that shorter LTL can reduce the risk of ALS indirectly based on IVs from a GWAS on 37,684 individuals of 
European ancestry $[28,29]$. The GWAS was a proxy for exposure measured LTL by quantitative PCR and the power of statistics was declared to be less than $30 \%$.

The exact underlying mechanism linking LTL to ALS is still unclear. Both leave-one-out analysis and single SNP analysis of two sets of IVs indicated that the OBFC1 (oligonucleotide/oligosaccharide-binding fold containing one) locus had a strong effect on ALS. The OBFC1 protein is part of the TPP1 protein complex that interacts with the telomerase and the telomere ssDNA-binding proteins, participates in maintaining telomere integrity and negatively regulates telomerase action [30-32]. Overexpression of truncated mutants in OBFC1 leads to telomere elongation in cancer cells [30], but no related studies have been performed in ALS. Because longer telomeres appeared to be a promising marker for the prognosis of ALS, extending the median survival time by $16 \%$ [6], we hypothesized that this genotype may be a protective indicator for disease and a predictor for the slow pattern of progression and that its function could be related to the onset and development of ALS. However, other clinical studies and zoological experiments are needed for further verification. It would be Four next step to investigate the relationship between OBFC1 and ALS diagnosis as well as prognosis and the function of OBFC1 in disease model mice and cells.

Furthermore, LTL is a solid marker for ageing [4]. Ageing is also regarded to share common pathologic pathways with ALS, which may provide ideas for the causality pathway between LTL and ALS. The transcriptomes of motor neurons differentiated from pluripotent stem cell of ALS patients are more similar to those of older neurons than those of motor neurons from age matched healthy controls [33]. Direct evidence has shown that the common C9orf72 hexanucleotide repeat expansion in ALS can form a stable G-quadruplex involved in the regulation of the telomere integrity and ageing [34]. Many ALS disease-causing genes, including OPTN, TBK1 and SOD1, play their important roles through the autophagy/lysosomal degradation pathway, which is shared with and vital in ageing [35]. Hence, the protective role of longer telomeres on ALS may be led by the increased cell proliferative activity and an enhanced ability to cope with oxidative stress, excitatory cytotoxicity, and apoptosis [36].

In addition, there may be other mechanisms that participate in the protective effect of LTL on ALS. Population-based research has demonstrated that telomere length displays sex differences [37], partly because oestrogen directly activates a promoter of telomerase [38] and enhances the activation of telomerase through the phosphoinositol-3-kinase/Akt [39] and nitric oxide pathways [40], leading to decelerated telomere shortening. According to our results, a longer telomere will decrease the risk of ALS, which is consistent with the fact that the prevalence of ALS in males is higher than that in females [41]. We deduced that oestrogen may further assist the role of telomeres in ALS. Oestrogen supplementation may have a positive effect on ALS. Animal experiments have proven that the extra 17 $\beta$-oestradiol (known as the most potent form of oestrogen) has a promising influence on ALS, which improved motor performance in male SOD1 G93A mice [42] and delayed the disease progression in ovariectomized mice to 137 days [43]. Although oestrogen replacement treatment is associated with attenuated motor symptoms in Parkinson's disease [44], high-quality clinical trials on ALS are still missing and in need to be carried out. Similar to supplements that slow telomere shortening, some effective habits to delay 
telomere shortening, such as lower stress and a high quality diet (e.g. the intake of $\omega-3$ free fatty acids, some antioxidants, and low consumption of saturated fat) [45] are also worth of trying in the further exploration of ALS treatment. Nonetheless, given the high consumption status and high metabolism of ALS patients, the impact of low intake of saturated fat is still unknown. However, it may provide new ideas for disease management and treatment in the future.

Our study has the following merits: (1) unitary race control; (2) including the largest current study to explore the causal relationship between LTL and ALS; (3) the heritability of exposure is impressive, and (4) MR analysis minimizes the interference of confounders and reverse causality. However, we still need to note some limitations: (1) the U-shaped relationship cannot be explained based on the principle of MR that the risk of disease is linearly related to telomere length; and (2) the relationship between sex, LTL and ALS also has been discussed. We cannot investigate the sex-specific effects of LTL on ALS, because of the absence of an available corresponding GWAS. Similarly, (3) the potential effect of LTL on the prognosis of ALS was deduced according to recent publications, but not verified with the MR approach due to the lack of relevant outcome data (clinical progression pattern, cognitive impairment, and survival).

\section{Conclusion}

Our study suggests that a longer LTL has a causal relationship with ALS in the European population mainly based on an LTL-related GWAS with 9190 individuals and underscores the importance of protecting against telomere loss in ALS.

\section{Abbreviations}

ALS: amyotrophic lateral sclerosis

leukocyte telomere length (LTL)

MR: Mendelian randomization

GWAS: genome-wide association study

IVW: inverse variance weighted method

IV: instrumental variable

SD: standard deviation

LD: linkage disequilibrium

Cl: confidence interval

BMI: body mass index 
SNP: single nucleotide polymorphism

OR: odds ratio

OBFC1: oligonucleotide/oligosaccharide-binding fold containing one

\section{Declarations}

\section{Ethics approval and consent to participate}

There were no patients directly involved in the overall process of our study. Our study is based on publicly available data only. All human studies included in this analysis were conducted according to the Declaration of Helsinki. Consent to participate is not applicable.

\section{Consent for publication}

All authors agreed to the publication of this article.

\section{Conflicts of interest}

The authors do not have any conflicts of interest to declare.

\section{Availability of data and materials}

All data generated or analyzed during this study are included in this published article and its supplementary information files.

\section{Code availability}

Codes generated or used during the study are available from the corresponding author by request.

\section{Acknowledgments}

We would like to thank for the GWASs for making the summary data publicly available, and we are grateful to all the participants who contributed to those studies. We also thank the National Natural Science Foundation of China (grant number: 81873784) for the support.

\section{Funding}

This study was supported by the National Natural Science Foundation of China (grant numbers: 81873784).

\section{Authors' contributions}

Dongsheng Fan designed the study, Tao Huang supervised the work, and Kailin Xia analysed the data and wrote the manuscript. Yajun Wang and Gan Zhang revised the draft. Linjing Zhang supervised data 
analysis.

\section{References}

1. Pandya VA, Patani R. Decoding the relationship between ageing and amyotrophic lateral sclerosis: a cellular perspective. Brain. 2019 Dec 18;143(4):1057-1072.

2. Arthur KC, Calvo A, Price TR, et al. Projected increase in amyotrophic lateral sclerosis from 2015 to 2040. Nat Commun. 2016 Aug 11;7:12408.

3. Haycock PC, Burgess S, Nounu A, et al. Association between telomere length and risk of cancer and non-neoplastic diseases: a Mendelian randomization study. JAMA Oncol. 2017 May;3(5)(1):636-51.

4. Kuo CL, Pilling LC, Kuchel GA, et al. Telomere length and aging-related outcomes in humans: a Mendelian randomization study in 261,000 older participants. Aging Cell. 2019 Dec;18(6):e13017.

5. Guo Y, Yu H. Leukocyte Telomere Length Shortening and Alzheimer's Disease Etiology. J Alzheimers Dis. 2019;69(3):881-5.

6. Al Khleifat A, lacoangeli A, Shatunov A, et al. Telomere length is greater in ALS than in controls: a whole genome sequencing study. Amyotroph Lateral Scler Front Degener. 2019 May;20(3-4):22934.

7. De Felice B, Annunziata A, Fiorentino G, et al. Telomerase expression in amyotrophic lateral sclerosis (ALS) patients. J Hum Genet. 2014 Oct;59(10):555-61.

8. Linkus $B$, Wiesner $D$, Messner $M$, et al. Telomere shortening leads to earlier age of onset in ALS mice. Aging (Albany NY). 2016 Feb;8(2):382-393.

9. Smith GD, Ebrahim S. 'Mendelian randomization': can genetic epidemiology contribute to understanding environmental determinants of disease? Int J Epidemiol. 2003 Feb;32(1):1-22.

10. Haycock PC, Burgess S, Wade KH, et al. Best (but oft-forgotten) practices: the design, analysis, and interpretation of Mendelian randomization studies. Am J Clin Nutr. 2016 Apr;103(4):965-78.

11. Mangino M, Hwang SJ, Spector TD, et al. Genome-wide meta-analysis points to CTC1 and ZNF676 as genes regulating telomere homeostasis in humans. Hum Mol Genet. 2012 Dec 15;21(24):53855394.

12. Kimura M, Stone RC, Hunt SC, et al. Measurement of telomere length by the Southern blot analysis of terminal restriction fragment lengths. Nat Protoc. 2010 2010/09/01;5(9):1596-1607.

13. Lai TP, Wright WE, Shay JW. Comparison of telomere length measurement methods. Philos Trans R Soc Lond B Biol Sci. 2018 Mar 5;373(1741):20160451.

14. Adams CD, Boutwell BB. A Mendelian randomization study of telomere length and blood-cell traits. Sci Rep. 2020 Jul;22(1):12223. 10(.

15. Pierce $B L$, Ahsan $H$, Vanderweele TJ. Power and instrument strength requirements for Mendelian randomization studies using multiple genetic variants. Int J Epidemiol. 2011 Jun;40(3):740-52.

16. Li C, Stoma S, Lotta LA, et al. Genome-wide association analysis in humans links nucleotide metabolism to leukocyte telomere length. Am J Hum Genet. 2020 2020/03/05/;106(3):389-404. 
17. Nicolas A, Kenna KP, Renton AE, et al. Genome-wide analyses identify KIF5A as a novel ALS gene. Neuron. 2018 Mar 21;97(6):1268-1283.e6.

18. Emdin CA, Khera AV, Natarajan P, et al. Genetic association of waist-to-hip ratio with cardiometabolic traits, type 2 diabetes, and coronary heart disease. JAMA. 2017 Feb;14(6):626-34. 317(.

19. Burgess S, Bowden J, Fall T, et al. Sensitivity Analyses for Robust Causal Inference from Mendelian Randomization Analyses with Multiple Genetic Variants. Epidemiology. 2017 Jan;28(1):30-42.

20. Bowden J, Davey Smith G, Haycock PC, et al. Consistent estimation in Mendelian randomization with some invalid instruments using a weighted median estimator. Genet Epidemiol. 2016 May;40(4):304-14.

21. Burgess S, Thompson SG. Interpreting findings from Mendelian randomization using the MR-Egger method. Eur J Epidemiol. 2017 May;32(5):377-89.

22. Verbanck M, Chen CY, Neale B, et al. Detection of widespread horizontal pleiotropy in causal relationships inferred from Mendelian randomization between complex traits and diseases. Nat Genet. 2018 May;50(5):693-8.

23. Hemani G, Tilling K, Smith GD. Orienting the causal relationship between imprecisely measured traits using GWAS summary data. PLoS Genet. 2017 Nov;13(11):e1007081.

24. Hemani G, Zheng J, Elsworth B, et al. The MR-Base platform supports systematic causal inference across the human phenome. Elife. 2018 May 30;7:e34408.

25. Niccoli T, Partridge L. Ageing as a risk factor for disease. Curr Biol. 2012 Sep 11;22(17):R741-R752.

26. Aviv A, Shay JW. Reflections on telomere dynamics and ageing-related diseases in humans. Philos Trans R Soc Lond B Biol Sci. 2018 Mar 5;373(1741):20160436.

27. Cawthon RM. Telomere measurement by quantitative PCR. Nucleic Acids Res. 2002 May 15;30(10):e47.

28. Codd V, Nelson CP, Albrecht E, et al. Identification of seven loci affecting mean telomere length and their association with disease. Nat Genet. 2013 Apr;45(4):422-427, 427e1-2.

29. Gao Y, Wang T, Yu X, et al. Mendelian randomization implies no direct causal association between leukocyte telomere length and amyotrophic lateral sclerosis. Sci Rep. 2020 Jul;22(1):12184. 10 (.

30. Wan M, Qin J, Songyang Z, et al. OB fold-containing protein 1 (OBFC1), a human homolog of yeast Stn1, associates with TPP1 and is implicated in telomere length regulation. J Biol Chem. 2009 Sep 25;284(39):26725-26731.

31. Levy D, Neuhausen SL, Hunt SC, et al. Genome-wide association identifies OBFC1 as a locus involved in human leukocyte telomere biology. Proc Natl Acad Sci U S A. 2010 May;18(20):9293-8. 107(.

32. Li S, Makovets S, Matsuguchi T, et al. Cdk1-dependent phosphorylation of Cdc13 coordinates telomere elongation during cell-cycle progression. Cell. 2009 Jan 9;136(1):50-61.

33. Ho R, Sances S, Gowing G, et al. ALS disrupts spinal motor neuron maturation and aging pathways within gene co-expression networks. Nat Neurosci. 2016 Sep;19(9):1256-67. 
34. Fratta P, Mizielinska S, Nicoll AJ, et al. C9orf72 hexanucleotide repeat associated with amyotrophic lateral sclerosis and frontotemporal dementia forms RNA G-quadruplexes. Sci Rep. 2012;2:1016.

35. Niccoli T, Partridge L, Isaacs AM. Ageing as a risk factor for ALS/FTD. Hum Mol Genet. 2017 Oct 1;26(R2):R105-r113.

36. Fu W, Killen M, Culmsee $C$, et al. The catalytic subunit of telomerase is expressed in developing brain neurons and serves a cell survival-promoting function. J Mol Neurosci. 2000;14(1):3-15. 2000/04/01.

37. Barrett ELB, Richardson DS. Sex differences in telomeres and lifespan [https://doi.org/10.1111/j.1474-9726.2011.00741.x] Aging Cell. 2011 2011/12/01;10(6):913-21.

38. Kyo S, Takakura M, Kanaya T, et al. Estrogen activates telomerase. Cancer Res. 1999 Dec 1;59(23):5917-5921.

39. Simoncini T, Hafezi-Moghadam A, Brazil DP, et al. Interaction of oestrogen receptor with the regulatory subunit of phosphatidylinositol-3-OH kinase. Nature. 2000 Sep;28(6803):538-41. 407(.

40. Grasselli A, Nanni S, Colussi C, et al. Estrogen receptor-alpha and endothelial nitric oxide synthase nuclear complex regulates transcription of human telomerase. Circ Res. 2008 Jul 3;103(1):34-42.

41. Xu L, Liu T, Liu L, et al. Global variation in prevalence and incidence of amyotrophic lateral sclerosis: a systematic review and meta-analysis. J Neurol. 2020;2020/04(01):267(4):944-53.

42. Heitzer $M$, Kaiser $S$, Kanagaratnam M, et al. Administration of $17 \beta$-estradiol improves motoneuron survival and down-regulates inflammasome activation in male SOD1(G93A) ALS mice. Mol Neurobiol. 2017;2017(12/01):54(10):8429-43.

43. Groeneveld GJ, Van Muiswinkel FL, Sturkenboom JM, et al. Ovariectomy and 17 $\beta$-estradiol modulate disease progression of a mouse model of ALS. Brain Res. 2004 2004/09/17/;1021(1):128-131.

44. Parkinson Study Group POETRY Investigators. A randomized pilot trial of estrogen replacement therapy in post-menopausal women with Parkinson's disease. Park Relat Disord. 2011;2011(12/01/):17(10):757-60.

45. Ornish D, Lin J, Daubenmier J, et al. Increased telomerase activity and comprehensive lifestyle changes: a pilot study. Lancet Oncol. 2008 2008/11/01/;9(11):1048-1057.

\section{Figures}




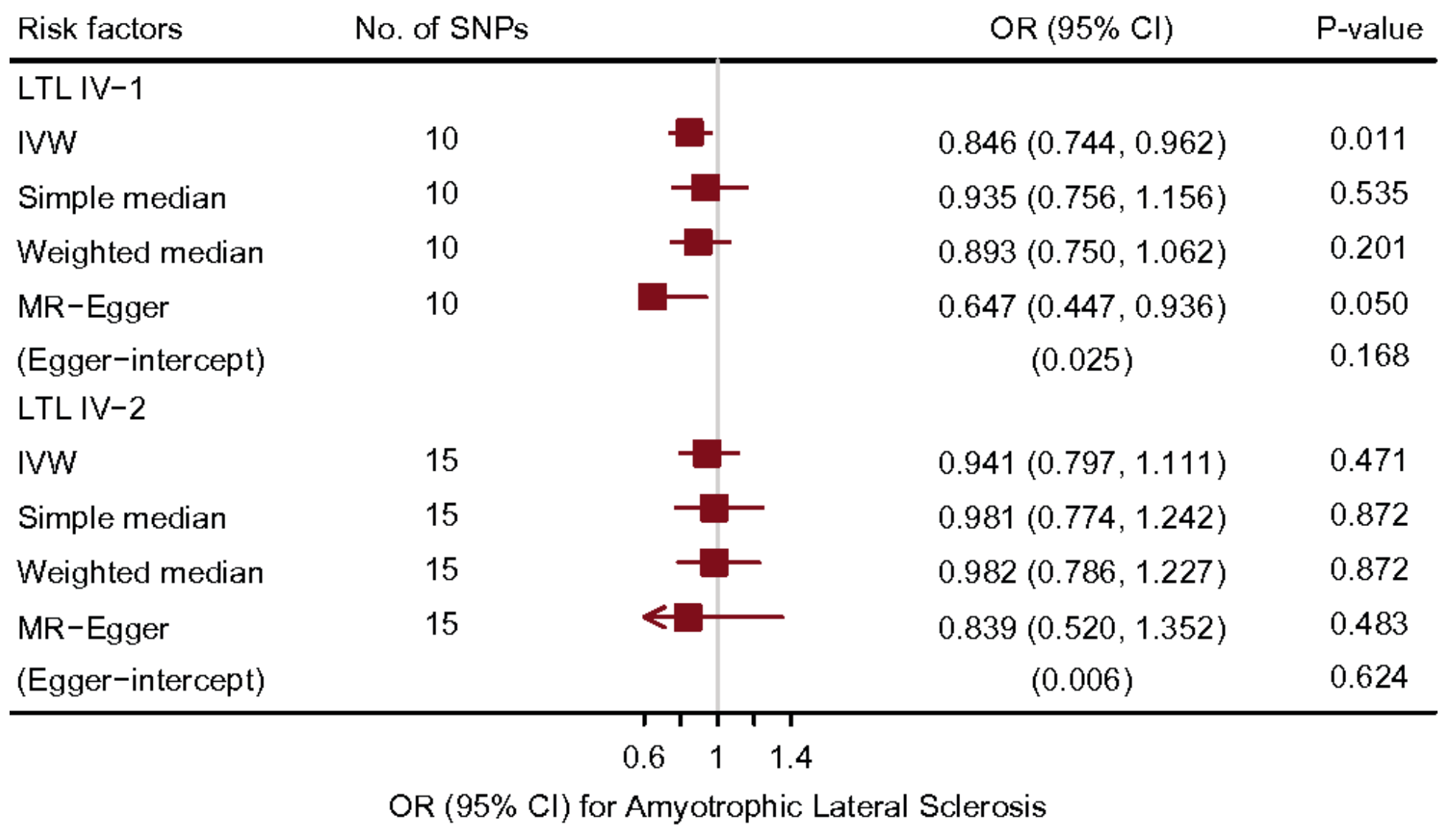

\section{Figure 1}

Association between genetically predicted leukocyte telomere length (LTL) and amyotrophic lateral sclerosis (ALS) Estimates are per approximately 1 standard deviation increase in leukocyte telomere length (LTL; bp). OR, odds ratio; $\mathrm{Cl}$, confidence interval; IVW, inverse variance weighted. 


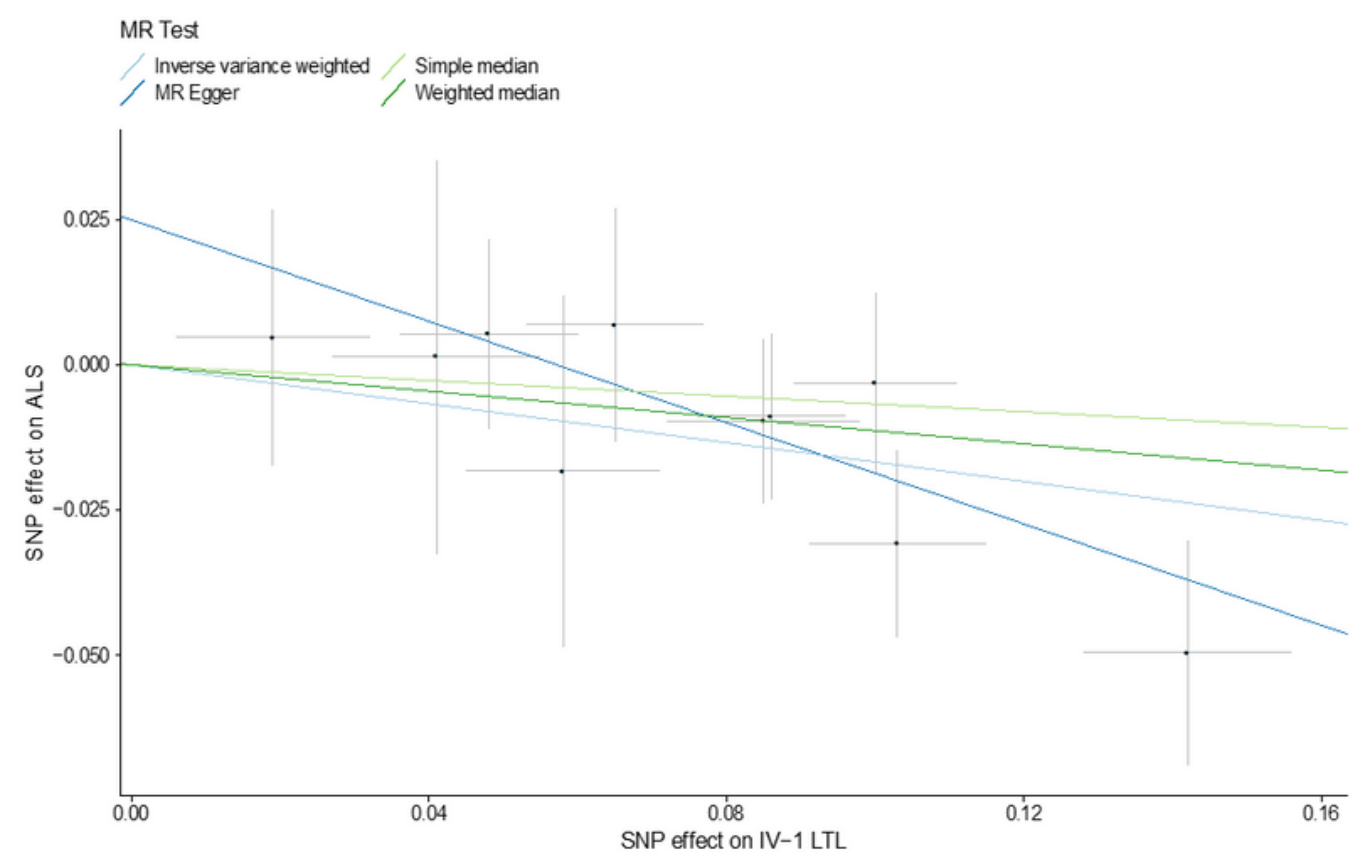

b

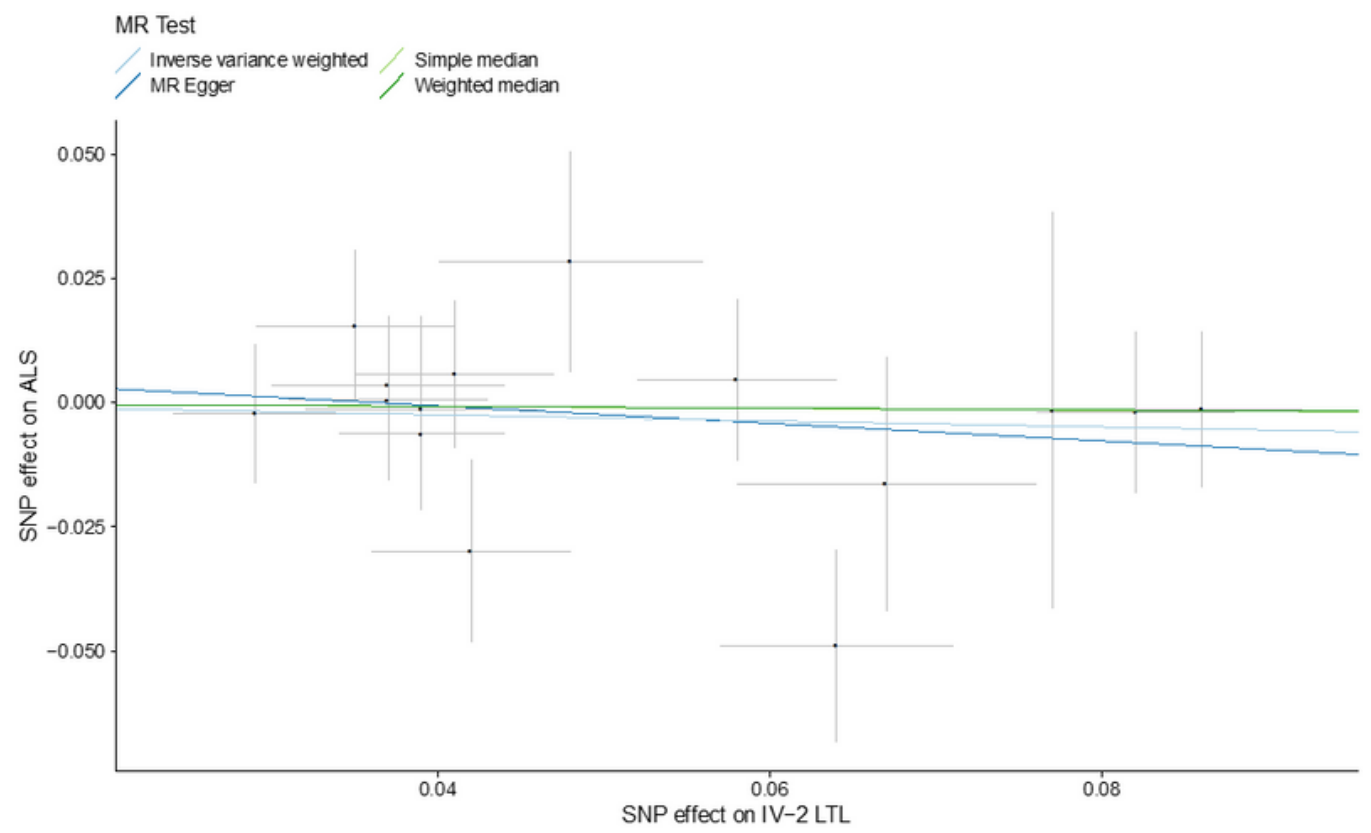

Figure 2

Scatter plot of single nucleotide polymorphism (SNP) effects on leukocyte telomere length versus amyotrophic lateral sclerosis (ALS), with the slope of each line corresponding to the estimated Mendelian randomization (MR) effect per method. a: results based on IV-1; b: results based on IV-2

\section{Supplementary Files}


This is a list of supplementary files associated with this preprint. Click to download.

- additionalfile.docx 